This item was submitted to Loughborough's Research Repository by the author.

Items in Figshare are protected by copyright, with all rights reserved, unless otherwise indicated.

\title{
Toward adaptive and intelligent electroadhesives for robotic material handling
}

PLEASE CITE THE PUBLISHED VERSION

http://dx.doi.org/10.1109/LRA.2016.2646258

\section{PUBLISHER}

(c) The Authors. Published by the IEEE.

\section{VERSION}

VoR (Version of Record)

\section{PUBLISHER STATEMENT}

This work is made available according to the conditions of the Creative Commons Attribution 3.0 Unported (CC BY 3.0) licence. Full details of this licence are available at: http://creativecommons.org/licenses/by/3.0/

\section{LICENCE}

CC BY 3.0

\section{REPOSITORY RECORD}

Guo, Jianglong, Thomas Bamber, Yuchen Zhao, Matthew Chamberlain, Laura Justham, and Michael R. Jackson. 2016. "Toward Adaptive and Intelligent Electroadhesives for Robotic Material Handling". figshare. https://hdl.handle.net/2134/24147. 


\title{
Toward Adaptive and Intelligent Electroadhesives for Robotic Material Handling
}

\author{
Jianglong Guo, Member, IEEE, Thomas Bamber, Student Member, IEEE, Yuchen Zhao, Student Member, IEEE, \\ Matthew Chamberlain, Laura Justham, and Michael Jackson
}

\begin{abstract}
An autonomous, adaptive, and intelligent electroadhesive material handling system has been presented in this paper. The system has been proposed and defined based on the identification of a system need through a comprehensive literature review and laboratory-based experimental tests. The proof of the proposed concept has been implemented by a low cost and novel electroadhesive pad design and manufacture process, and a mechatronic and reconfigurable platform, where force, humidity, and capacitive sensors have been employed. This provides a solution to an autonomous elelctroadhesive material handling system that is environmentally and substrate material adaptive. The results have shown that the minimum voltage can be applied to robustly grasp different materials under different environment conditions. The proposed system is particularly useful for pick-and-place applications where various types of materials and changing environments exist such as robotic material handling applications in the textile and waste recycling industry.
\end{abstract}

Index Terms-Adaptive and intelligent electroadhesive, electroadhesion, electroadhesive design and manufacture, grippers and other end-effectors, material handling, sensor-based control.

\section{INTRODUCTION}

$\mathbf{E}$ LECTROADHESION is an electrically controllable attractive effect between an electroadhesive pad and a substrate [1], [2]. Electroadhesion has been extensively used as an advanced adhesion mechanism for several robots [3]-[8] and material handling applications [9]-[11] due to the fact that electroadhesion, compared with other adhesion mechanisms [12], has several distinctive advantages including enhanced adaptability, reduced complexity, flexible and gentle handling, and ultra-low energy consumption [13].

An electroadhesion system usually contains four essential components: the electroadhesive pad, the high voltage power source, the substrate, and the control system [14], as shown in Fig. 1. The electroadhesive pad contains conductive electrodes

Manuscript received September 14, 2016; accepted December 14, 2016. Date of publication December 18, 2016; date of current version January 16, 2017. This paper was recommended for publication by Associate Editor H. Ding and Editor A. Bicchi upon evaluation of the reviewers' comments. This work was supported by the EPSRC Centre for Innovative Manufacturing in Intelligent Automation under Grant EP/IO33467/1.

The authors are with the EPSRC Centre for Innovative Manufacturing in Intelligent Automation, Loughborough University, Loughborough LE11 3TU, U.K. (e-mail: J.Guo@lboro.ac.uk; T.Bamber@lboro.ac.uk; Y.Zhao@ lboro.ac.uk; M.R.Chamberlain@lboro.ac.uk; L.Justham@lboro.ac.uk; M.R. Jackson@lboro.ac.uk).

Color versions of one or more of the figures in this paper are available online at http://ieeexplore.ieee.org.

Digital Object Identifier 10.1109/LRA.2016.2646258

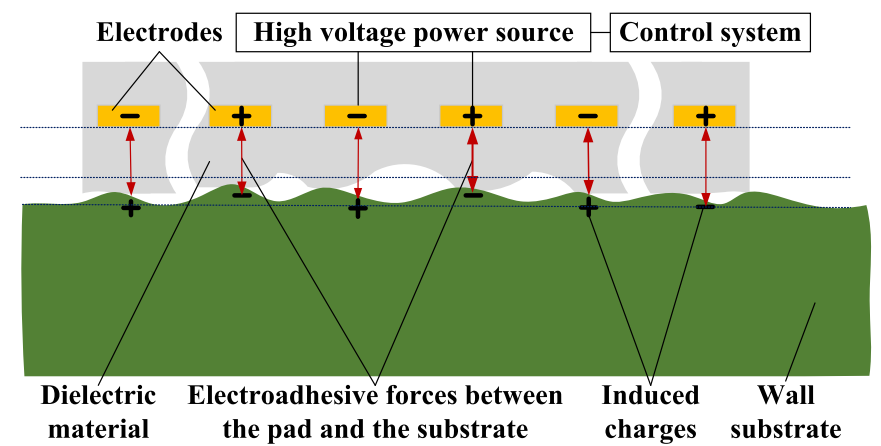

Fig. 1. Cross-sectional view of an electroadhesion system.

embedded in dielectric materials. The conductive electrodes are connected with high voltage power sources, usually in the range of $\mathrm{kV}$. The voltage source can be monopolar, bipolar, or multipolar, and based upon direct or alternating current. The dielectric materials are useful for preventing the charge neutralization and dielectric breakdown. The control system controls the on and off of the electroadhesion system.

Electroadhesion is a multidisciplinary, complicated, and dynamic electrostatic attraction phenomenon with over 33 variables influencing the electroadhesive forces obtainable between the electroadhesive pad and the substrate [11]. Pad geometry plays an important role in achieving the electroadhesive forces [14]-[16]. Both the pad geometry and voltage source type determine the types of substrate material to be picked up, and the pick-up and release speed.

The electroadhesive forces vary significantly on the same substrate with different surface textures [11]. Hybrid adhesion mechanisms (a combination of electroadhesion and dry adhesion) [17], fluid dielectrics attaching to the pad [18], a layer of elastic foam attaching to the pad [18], and inductive fibers [19] have been investigated to enable the electroadhesive pads to conform to different surface conditions. Flexible electroadhesive pads are able to conform to curved surfaces to some extent if compliant dielectric materials are used [15]. In order to grasp uncooperative objects/surfaces, however, morphing electroadhesive gripper mechanisms [20], curved electroadhesive gripper mechanisms [21], and vacuum augmented electroadhesive mechanisms [22] are more preferable.

Electroadhesion can enable the pad to adhere to different substrate materials such as smooth aluminium plates and rough concrete surfaces [24]. The electroadhesive forces, however, 
vary significantly on different substrate and dielectric materials [15], [17]. Sufficient voltage should therefore be applied to enable the pad to grasp different materials at the same time. Adhesion failure occurs when changing from grasping insulating materials to conductive materials as dielectric breakdown occurs when a high voltage is applied to conductive substrates. It is therefore desirable to have an optimized and intelligent electroadhesive system that can produce large enough forces when grasping different materials using lower voltages.

Unstable and unpredictable electroadhesive forces can be obtained in changing ambient environments where temperature, humidity, and air pressure change significantly during the day and between days [14], [23]. Designing and implementing an electroadhesive system that can output repeatable and reliable electroadhesive forces in changing ambient environments is therefore also highly desirable.

Current electroadhesion pads bring a certain level of adaptability. This is limited without embedding intelligence into the electroadhesion system. An adaptive and intelligent electroadhesion system is therefore needed and has been defined in this paper as an electrically controllable adhesion system that is adaptive to substrate surface texture, substrate shape, substrate material, one that is environmentally stable or adaptive to a changing environment with quick pick-up and release speeds, lower power consumption, longer life cycles, and greater safety.

Substrate surface texture and substrate shape adaptability have been investigated and implemented previously, as shown in a number of studies [17]-[22]. In this paper, the focus has been on the investigation into an environmentally and substrate material adaptive electroadhesion system, which is a novel approach not previously considered. Experimental validation of the need for this has been conducted and presented in Section II. The design and implementation of an autonomous, adaptive and intelligent electroadhesive material handling system have been completed and described in Section III. Conclusions and future work have been made and can be seen in Section IV.

\section{EXPERIMENTAL VALIDATION OF THE NEED FOR ADAPTIVE AND INTELLIGENT ELECTROADHESIVES}

\section{A. Principle of Electroadhesion}

For conductive substrates, the electroadhesive forces are generated mainly by electrostatic induction. For insulating substrates, the electroadhesive forces are generated mainly by electric polarizations [15], [24]. On conductive substrates, the electroadhesive forces obtainable can be derived by:

$$
F_{z}=F_{\text {Coulomb }}+F_{J-R}
$$

where $F_{\text {Coulomb }}$ denotes the Coulomb forces andc denotes the J-R electroadhesive forces [14].

The electroadhesive forces generated on insulating substrates can be expressed by [14]:

$$
F_{z}=P_{\text {polarization }} E
$$
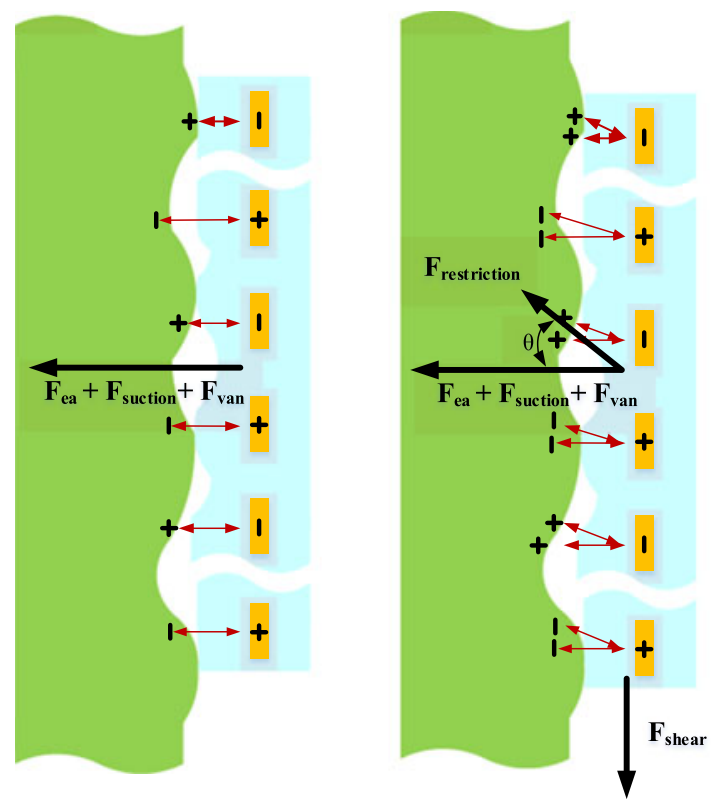

Fig. 2. The analysis of the electroadhesive force analysis in normal and shear before and after applying the shear force (the weight of the pad is neglected) [14].

where $E=\left\{\begin{array}{cc}0, & t=0 \\ E, & t>0\end{array}\right.$ and $P_{\text {polarization }}=P_{e}+P_{i}+P_{o}+P_{d}+$ $P_{s}+P_{\text {others }}$, with $P_{e}$ is the electronic polarization, $P_{i}$ is the ionic polarization, $P_{o}$ is the orientation polarization, $P_{d}=$ $P_{\text {hop }}+P_{\text {face }}$ is the space charge polarization which includes the hopping polarization, $P_{\text {hop }}$ and the interfacial polarization, $P_{\text {face }}, P_{s}$ is the spontaneous polarization, and $P_{\text {others }}$ is other types of polarizations such as the nomadic polarization.

All the former results have shown that the shear electroadhesive forces are larger than that of the normal forces. Most of them have not, however, described the suction forces (if not tested in vacuum) and Van Der Waals forces. The inclusion of the suction forces due to the air gap and Van Der Waals forces due to the intimate contact between the electroadhesive pad and the substrate can be seen in Fig. 2. The actual normal electroadhesive forces obtainable can therefore be expressed as:

$$
F_{\text {normal }}=F_{\text {ea }}+F_{\text {suction }}+F_{\text {van }}
$$

where $F_{\text {ea }}$ is the normal electroadhesive forces, $F_{\text {suction }}$ is the suction forces between the pad and the substrate, and $F_{\text {van }}$ is the Van Der Waals forces.

When a shear force is applied on the pad, restriction forces occur, as shown in Fig. 2(b) [14]. Assuming that there is an angle, $\theta$, between the forces in normal and the restriction forces, the shear electroadhesive forces obtainable can then be expressed as:

$$
\begin{aligned}
F_{\text {shear }}= & \mu\left(F_{\text {ea }}+F_{\text {suction }}+F_{\text {van }}+F_{\text {restriction }} \cos \theta\right) \\
& +F_{\text {restriction }} \sin \theta
\end{aligned}
$$

It is therefore inappropriate to derive the normal electroadhesive forces by the division of the measured shear forces and friction coefficients as it is difficult to quantify the restriction forces, suction forces, and Van Der Waals forces [14], Although 


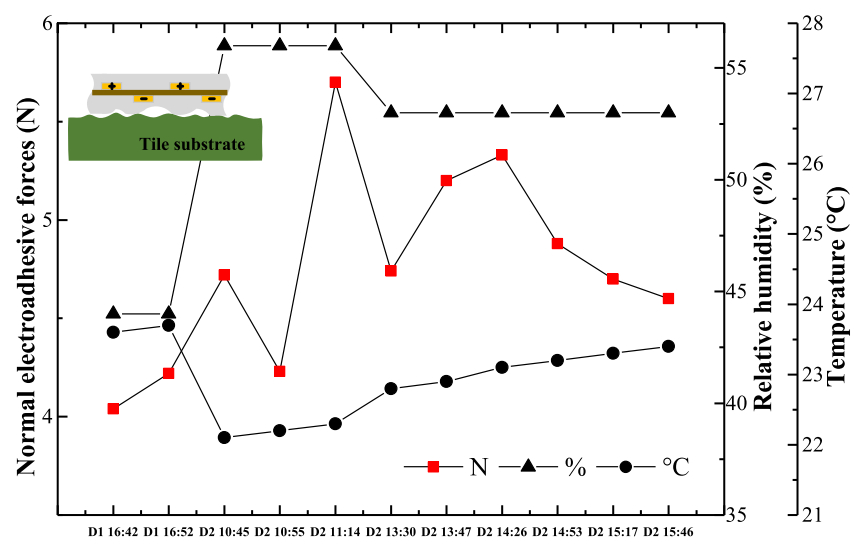

Fig. 3. The electroadhesive forces obtained in different ambient environmental conditions over a two-day period.

the shear electroadhesive forces are usually larger than the normal forces, due to the fact that sheer forces cannot be precisely evaluated, all the tests completed in this paper have been based on the normal direction.

\section{B. Influence of Environmental Factors}

Environmental factors affect and modify the dielectric properties (such as dielectric strength, permittivity, and resistivity) of the electroadhesive pads. Also, they arouse electric discharges and dielectric degradation that cause the failure of the adhesion [14]. Within the research laboratory where our fundamental research has been undertaken, the lab temperature has changed from 17 to $28^{\circ} \mathrm{C}$, relative humidity changed from 28 to $73 \%$ and air pressure changed from 996.3 to $1015.2 \mathrm{hPa}$ between January and August 2015, recorded by a weather station [14]. In order to understand how these environmental changes were affecting the normal adhesion force on the electroadhesive pad, a two-day test was conducted in the ambient environment when variables were controlled except the temperature $\left(22.1-23.7^{\circ} \mathrm{C}\right)$, relative humidity $(44-56 \%)$ and air pressure $(1002-1009 \mathrm{hPa})$. A professionally manufactured double-sided electroadhesive pad and a tile substrate was used. The polyimide coverlay side of the pad was facing the substrate when measuring the electroadhesive forces. Results shown in the Fig. 3 demonstrate that the electroadhesive forces do vary in the changing ambient environment. The results from a further five-day period have also been obtained when variables have been maintained, except the temperature $\left(20.8-21.5^{\circ} \mathrm{C}\right)$, relative humidity $(43-64 \%)$ and air pressure $(993.8-1013 \mathrm{hPa})$ [14]. Both results have indicated that humidity is the most significant environmental factor influencing the electroadhesive forces obtainable. Please note that the electroadhesive force testing platform and procedure for the result presented in Fig. 3 were based on the same force test rig and process published by Guo et al., where a 6-axis ATI gamma force/torque (F/T) sensor, with a tolerance of $\pm 0.05 \mathrm{~N}$, was used [11].

The above results indicate that it is necessary to design and manufacture environmentally adaptive and stable electroadhesives. This could be done by designing novel pads using novel

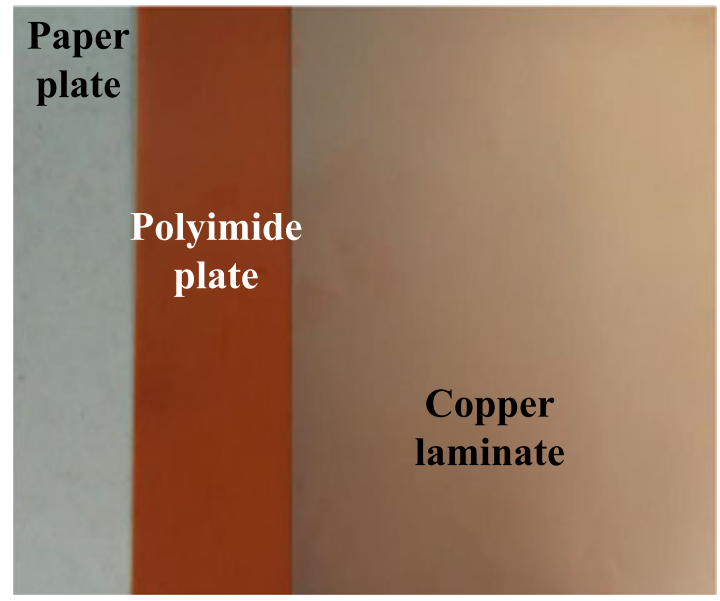

Fig. 4. The paper plate, PI plate and copper laminate.

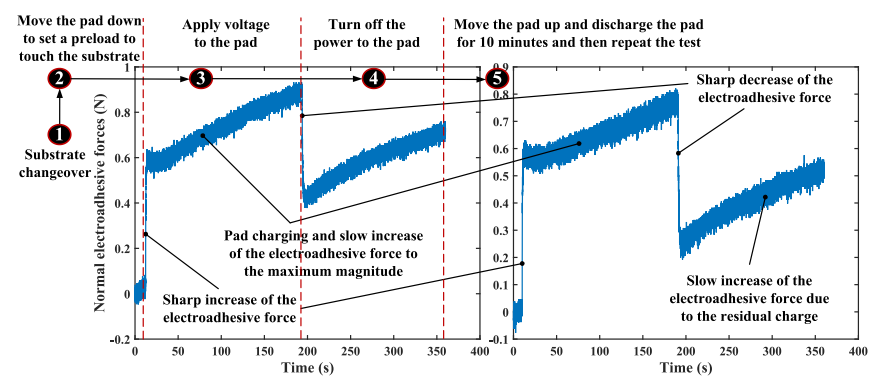

Fig. 5. The electroadhesive force testing procedure to check the influence of the substrate materials.

materials that are less sensitive to environmental variations and can produce stable electroadhesion properties in changing environments. An alternative method is to inform the electroadhesion system of the environmental change based on humidity and temperature sensors and then adjust the applied voltage accordingly. The latter method has been adopted in this paper.

\section{Influence of Substrate Materials}

An experiment testing procedure was designed and employed to test the three A4 size substrate materials used in this paper: a paper plate, a polyimide (PI) plate, and a copper laminate (see Fig. 4). The testing procedure, taking the copper laminate as an example, can be seen in Fig. 5 and has been based on the platform shown in Fig. 13. The pad has been charged on the three materials for three minutes' and then the power has been turned off but the forces have been recorded for another three minutes. The measurement results of the PI plate and paper plate can be seen in Figs. 6 and 7. The tests were conducted when the temperature was $22.7 \pm 0.1{ }^{\circ} \mathrm{C}$, relative humidity was $53 \pm 1 \%$, and pressure was $1011.3 \pm 0.2 \mathrm{hPa}$. Electroadhesion is a dynamic electrostatic attraction phenomenon both on conductive and insulating substrates [11], [25]. The dynamic polarization and depolarization time can be extremely long (such as hours) for some insulating substrates [14]. A different result obtained on a polycarbonate (PC) plate can be seen in Fig. 8. 


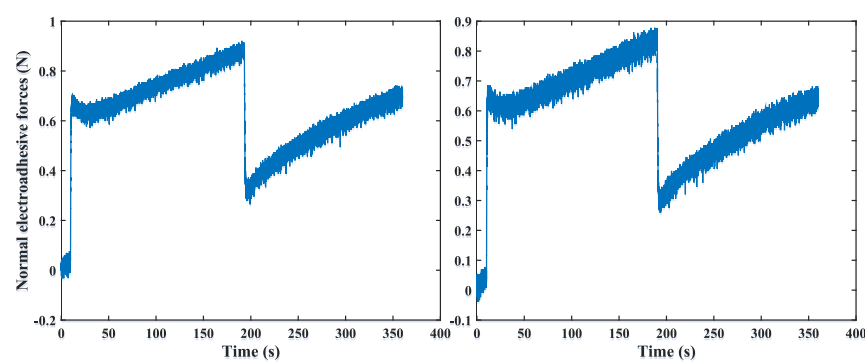

Fig. 6. The measurement result of the PI plate.

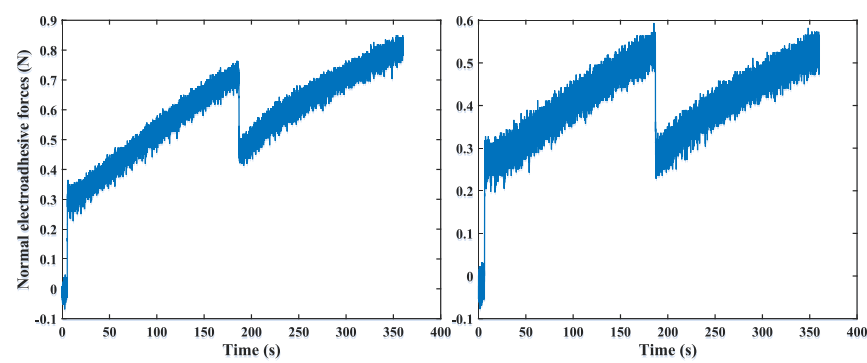

Fig. 7. The measurement result of the paper plate.

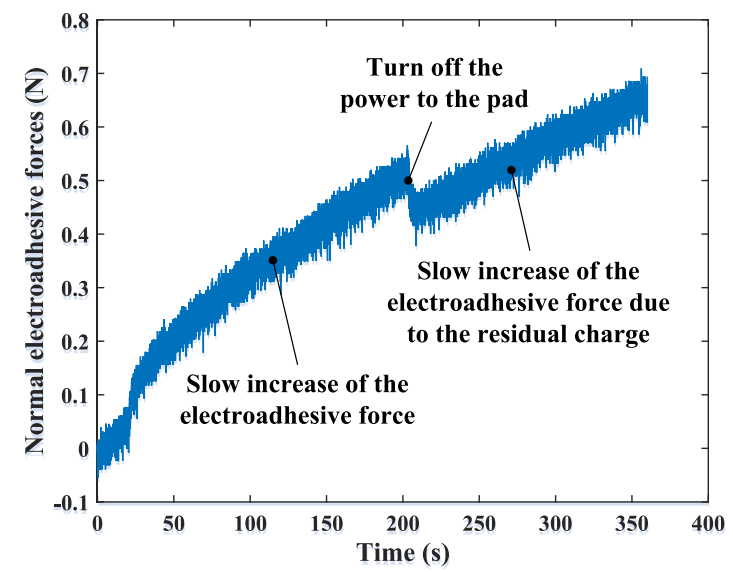

Fig. 8. The measurement result of the PC plate.

In this paper, only the paper plate, copper laminate, and PI plate, which have relatively quicker electroadhesion response properties, have been used for the proof of the proposed concept. A comparison the first test of three materials can be seen in Fig. 9.

The electroadheisve forces do vary between the pad and different substrate materials. It has also been shown in the supplementary video that different voltages should be applied to pick up the paper plate, PI plate, and copper laminate successfully. A higher voltage magnitude is required to pick up the paper. If a high voltage is used on all substrates, then the number of life cycles of the pads would decrease and the power consumption would increase due to higher magnitude of polarization of the material. Also, adhesion failure would occur when changing from picking up the paper and PI plate to the copper side of the copper laminate when using the high voltage. This is because

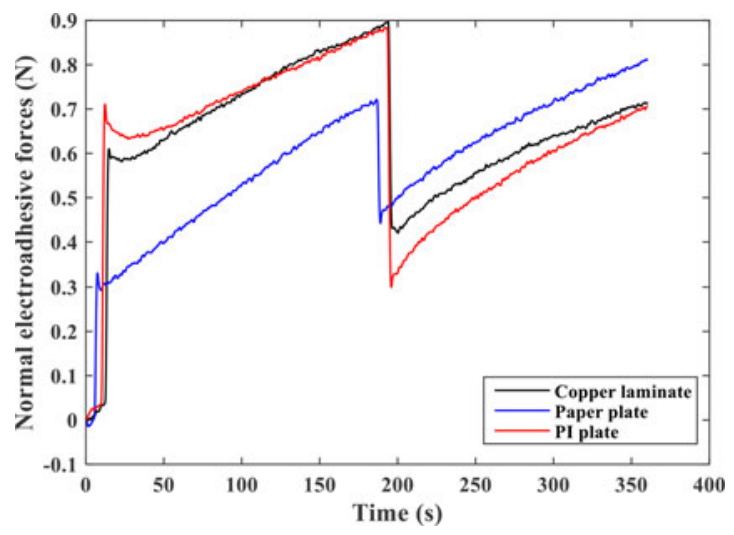

Fig. 9. The comparison of the measurement results of the paper plate, PI plate, and copper laminate.

the high voltage may induce discharge and dielectric breakdown of the pad.

Material identification or differentiation is therefore needed so that different minimum voltages can be applied on different materials. The electroadhesive pad itself is able to differentiate different materials based on the results shown in Figs. 8 and 9. This force sensor based method, however, is slow for industrial material handling applications as it takes several minutes to stabilize the preload and measure the forces. Novel control methods have been used to make the electroadhesive pad have two modes: the materials identification mode and the electroadhesive grasping mode [26].

In order to prove the proposed concept in a cost-effective way, an analog capacitive sensor was used to differentiate different materials and inform the electroadhesion system if there is a change of substrate material in a quicker way [26]. The output current of the capacitive sensor is 4-20 mA and the measuring range is $0-8 \mathrm{~mm}$. It has to be noted that there are some limitations associated with this capacitive sensor. A preliminary feasibility study has been conducted based on the Arduino setup [26]. The results have shown that the capacitive sensor cannot differentiate different smooth metals. Also, the capacitive sensor has been tested on the same four aluminum plates and three sandpaper samples used by Guo et al. [11] to investigate the influence of surface roughness to the result. For all the aluminium plates, the results have been the same, $0.85 \mathrm{~V}$. For the P1200 and P400 sandpaper samples, the result has been $1.35 \mathrm{~V}$. For the P120 sandpaper sample, however, the result has been $1.5 \mathrm{~V}$. This indicates that, if the surface roughness difference of the substrate is small, the capacitive sensor is insensitive to different surface textures. It is difficult for the capacitive sensor to identify the same material if the surface roughness difference of the substrate is large.

\section{IMPLEMENTATION OF THE ADAPTIVE AND INTELLIGENT ELECTROADHESION SYSTEM}

\section{A. Electroadhesive Pad Design and Manufacture}

A low-cost and novel in-house electroadhesive pad design and manufacturing process has been employed to produce the pad 


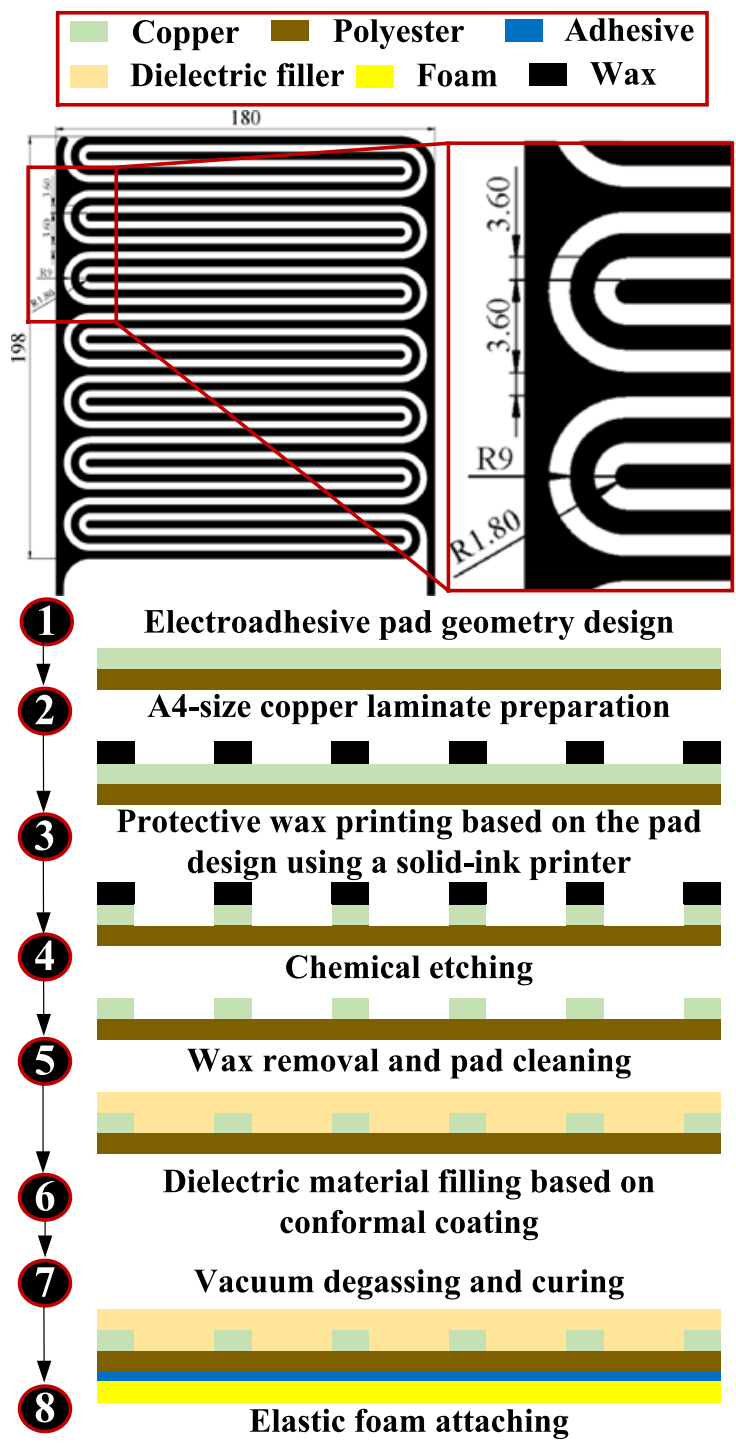

Fig. 10. The electroadhesive pad design and manufacture process.

for this study. This process has been based on the pad geometric design in Solidworks, solid-ink printing based on the design, wax removal, chemical etching, dielectric conformal coating, vacuum degassing and curing, and elastic foam attaching, as shown in Fig. 10.

The pad geometry has been based on a novel coplanar 'wormcomb' shape as it has shown improved performance in achieving forces over other pad designs [16]. The effective pad area has been made to be $180 \mathrm{~mm}$ (width) $\times 198 \mathrm{~mm}$ (length), and the electrode width and space between electrodes have been both set to $3.6 \mathrm{~mm}$. The A4 size copper laminate has been made of a $20 \mu \mathrm{m}$ copper adhered on a $23 \mu \mathrm{m}$ Polyester (PET, dielectric strength: $310 \mathrm{kV} / \mathrm{mm}$, dielectric constant: 3.2 ). VytaFlex urethane rubber has been used as the dielectric material. After the pad has been degassed and cured, a $10 \mathrm{~mm}$ layer of elastic foam has been adhered to the pad so that the pad can conform to different surface conditions.

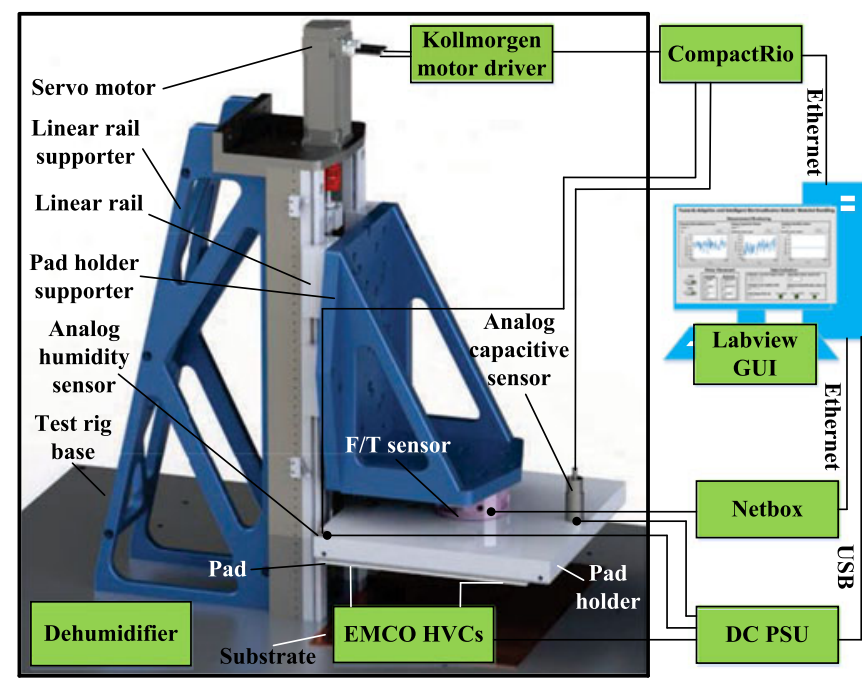

Fig. 11. The system diagram of the adaptive and intelligent electroadhesive robotic material handling system.

\section{B. System Integration for the Proposed Concept}

A bespoke mechatronic and reconfigurable electroadhesive material handling platform has been implemented to prove the concept proposed in this paper of an adaptive and intelligent electroadhesion system. The system diagram can be seen in Fig. 11, where the pad was connected with two EMCO high voltage converters (HVCs) with $( \pm) 0-10 \mathrm{kV}$ output and $0-5 \mathrm{~V}$ reference input. The reference input was from a direct current power supply unit (DC PSU). The communication between the DC PSU and the computer through was via a USB cable. The voltage difference applied on the pad was 4 times the assigned value minus 0.1 in Labview.

The pad was assembled on the pad holder, which was then connected with a 6-axis ATI Gamma Force/Torque $(F / T)$ sensor (resolution: $\pm 0.05 \mathrm{~N}$ ). The $\mathrm{F} / \mathrm{T}$ sensor, assembled on the pad hold supporter, was used to record the electroadhesive forces. A Netbox was used to enable the communication between the $F / T$ sensor and the computer via an Ethernet cable. The pad on the linear rail can achieve vertical movement using a servo motor (with an encoder) driven by a Kollmorgen motor driver connected with a CompactRio. The smallest movement of the linear rail that the encoder can detect was approximately $0.8 \mu \mathrm{m}$. The communication between the CompactRio and the computer through was via an Ethernet cable. The analog capacitive sensor and humidity have both been powered by the DC PSU and controlled by the computer via the CompactRio.

The physical platform can be seen in Fig. 11. A Labview interface has been developed for the interactive control of the movement of the linear rail and the supply voltage for the pad, and recording, displaying and saving the electroadhesive force data, the analog capacitive sensor output data, and the analog humidity sensor output data.

\section{Control Flowchart for the Proposed System}

An autonomous electroadhesive material handling process has been designed, as can be seen in Fig. 12, to prove the 


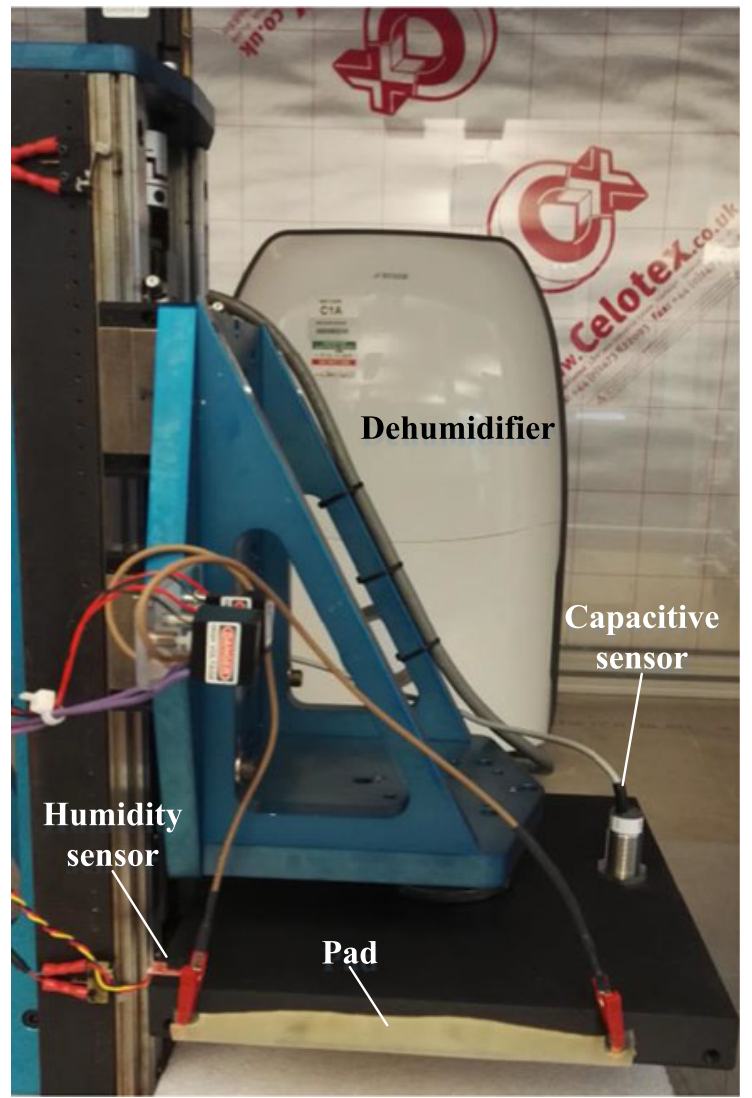

Fig. 12. The physical setup of the adaptive and intelligent electroadhesive robotic material handling system.

proposed concept. The only two manual steps are placing the substrate underneath the pad as the start and clicking the start button of the Labview program. The $F / T$ sensor, humidity sensor, and capacitive sensor are turned on after starting the Labview main program. Also, the motor moves down to touch the substrate and it stops until the force is equal and greater than $30 \mathrm{~N}$. This preload is useful to make sure the pad fully conforms the substrate surface and the capacitive is touching the substrate. The motor forward movement velocity has been set as $30 \mathrm{mms}^{-1}$, whereas the backward movement velocity has been $10 \mathrm{mms}^{-1}$. The motor movement acceleration has been set as $10 \mathrm{revs}^{-2}$. The selected values for the motor movement are for demonstration purposes and can be changed.

The material identification is then conducted by the capacitive sensor. Note that, for each substrate material, a priori capacitive testing and the minimum voltage needed to pick up the substrate material have been carried out. Then, a look-up table (LUT), linking the minimum voltage and the capacitive reading, can be generated. This LUT has then been built into the Labview program to achieve the automatic material identification. The limitation of this method is that the proposed system may not pick up the materials that are not included in the LUT.

After this, the humidity level of the surrounding environment is detected by the humidity sensor. Since an empirical relationship between the humidity level and the electroadhesive forces obtainable has been built into the Labview program, the HVCs

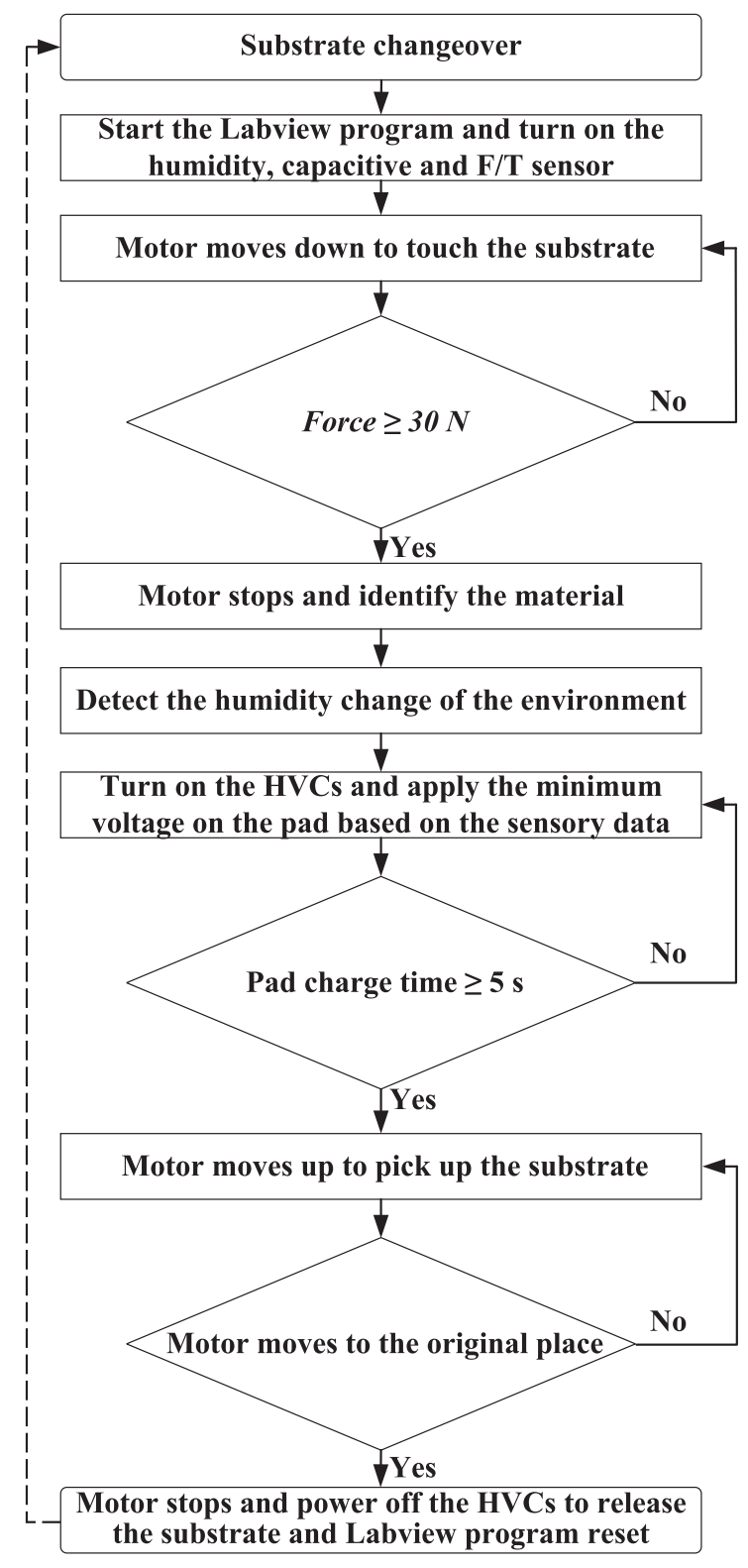

Fig. 13. The control flowchart for the adaptive and intelligent electroadhesive robotic material handling system.

are then turned on and assigned a value based on the result of the material identification and the humidity level to charge the pad for 5 seconds. Note that, for the prove of the proposed concept, only the empirical relationship between the varied humidity levels between $55 \%$ and $65 \%$, with a step of $1 \%$, and the paper plate substrate has been built into the Labview program. After 5 seconds, the motor moves back and the substrate is picked up by the pad based on the minimum voltage needed. The motor stops when it goes back to its original position and then the HVCs are turned off to release the substrate and the Labview program is reset for the next adaptive and intelligent electroadhesive material handling process. The 5 seconds' pad charge time and the material identification time are used for demonstrate purposes and can be changed to smaller values such as 1 second. 


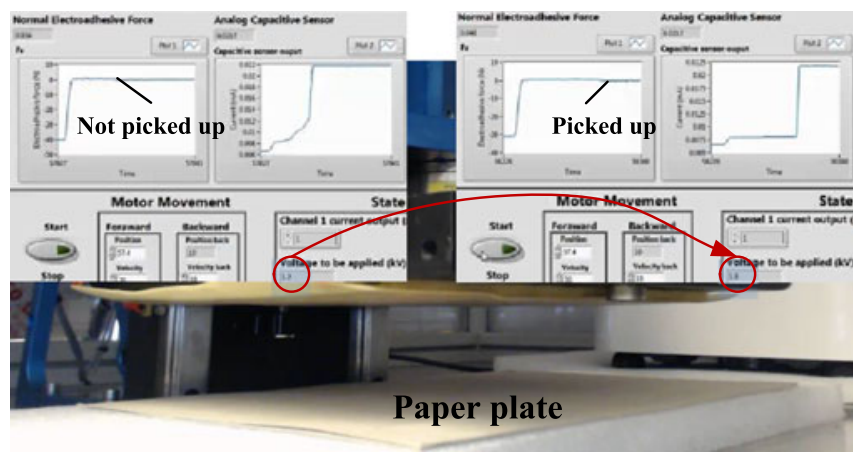

Fig. 14. The detection of the humidity change and automatic voltage adjustment to robustly pick up the paper plate.

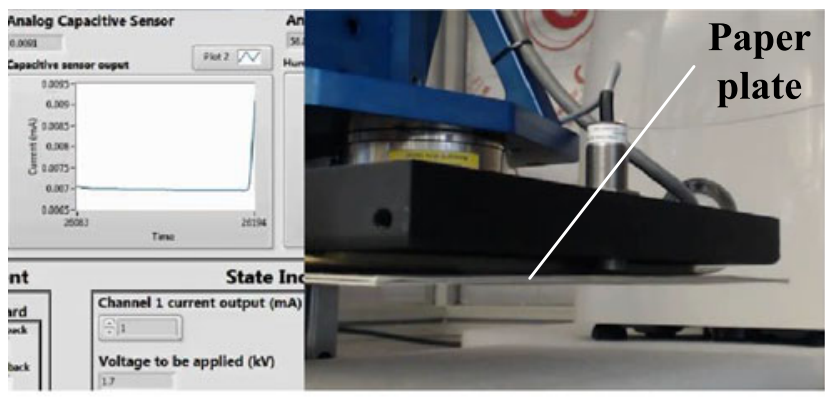

(a)

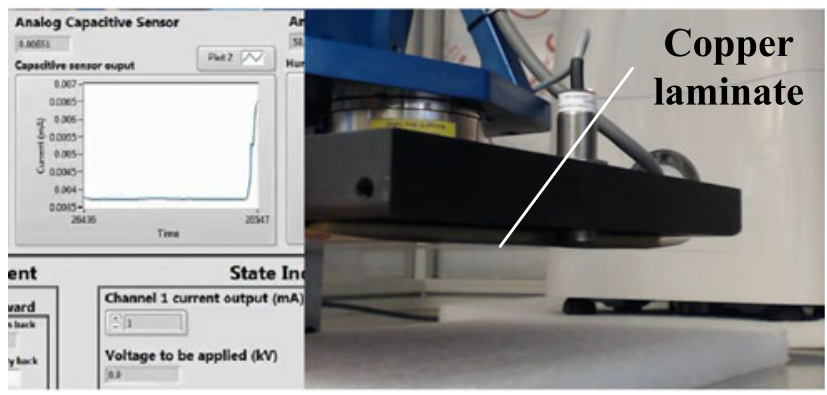

(b)

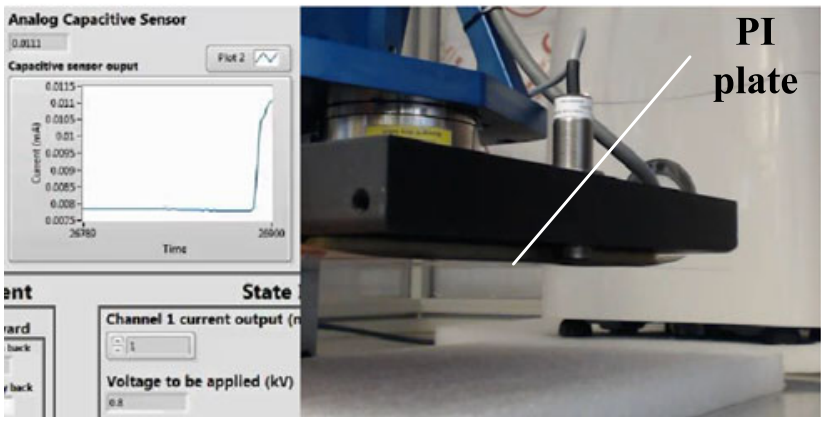

(c)

Fig. 15. The adaptive and intelligent electroadhesive system to picking up the paper plate, copper laminate, and the PI plate when the relative humidity was $58 \pm 1 \%$.

\section{CONCLUSION}

An autonomous, adaptive and intelligent electroadhesive material handling system has been implemented and demonstrated in this paper based on a low-cost and novel electroadhesive pad design and manufacture process, a mechatronic and recon- figurable platform with the integration of an $F / T$ sensor, an analog humidity sensor, and an analog capacitive sensor. The proposed system has been able to detect the change of the humidity level. When the relative humidity changed from $58 \pm 1 \%$ to $63 \pm 1 \%$ and was detected by the humidity sensor, a change of the minimum applied voltage $(6.4 \mathrm{kV}$ to $7.2 \mathrm{kV})$ was automatically conducted by the proposed system to pick up the paper plate successfully, as shown in Fig. 14. The proposed system has also been able to successfully pick up the paper plate, copper laminate, and PI plate, as can be seen in Fig. 15, by applying the minimum voltage $(6.4 \mathrm{kV}, 3.2 \mathrm{kV}$, and $2.8 \mathrm{kV}$ respectively) based on the sensory data.

We are still in the process of understanding this promising dynamic electroadhesion phenomenon, and we believe the proposed solution presented in this paper will bring a paradigm shift toward future electroadhesive material handling applications. Although the proposed solution is promising to enable an environmentally adaptive and substrate material adaptive elelctroadhesion material handling system, several limitations of this solution still exist. The limitations of the capacitive sensor have been described in part $\mathrm{C}$ of Section II. An analog inductive sensor can be used and integrated into the system in the future to differentiate different metals. In addition, a priori knowledge of the weight of different substrate materials and testing of the required minimum voltage should be embedded into and inform the proposed system as different voltage magnitude is required for the same material but has different weights. Furthermore, the manual feeding of the substrate materials has been used in this paper. This is because the proposed system has only one degree of freedom (DoF).

The main contributions of this paper include: 1) the propose of the adaptive and intelligent electroadhesion concept, based on a comprehensive literature survey and experimental results; 2) the new experimental procedure to investigate the relationship between the electroadhesive forces and different substrate materials and the results; 3 ) the addition of an elastic foam layer to the cost-effective electroadhesive pad design and manufacture process; 4) the system integration of an $F / T$ sensor, an analog humidity sensor, and an analog capacitive sensor to prove the proposed autonomous, adaptive and intelligent electroadhesive material handling system; 5) the control method of the proposed system based on sensory data and empirical relationships. Future work will include using a 6DOF robot for electroadhesive material handling. In addition, since the shear electroadhesive forces, although difficult to quantify, are usually larger than the normal forces, electroadhesive material handling applications based on the shear direction will be considered. Furthermore, the LUTs for the proposed system should be expanded, thus making the system more robust and adaptable to more substrate materials and environment conditions.

\section{REFERENCES}

[1] R. P. Krape, "Applications study of electroadhesive devices" National Aeronautics and Space Administration, Washington, DC, USA, NASA Contractor Report: NASA CR-1211, 1968.

[2] K. Rahbek, "Electroadhesion apparatus," U.S. Patent 2025 123, 1935. 
[3] A. Yamamoto, T. Nakashima, and T. Higuchi, "Wall climbing mechanisms using electrostatic attraction generated by flexible electrodes," in Proc. 2007 Int. Symp. Micro-NanoMechatronics. Human Sci., 2007, pp. 389-394.

[4] H. Prahlad, R. Pelrine, S. Stanford, J. Marlow, and R. Kornbluh, "Electroadhesive robots-wall climbing robots enabled by a novel, robust, and electrically controllable adhesion technology," in Proc. 2008 IEEE Int. Conf. Robot. Autom., 2008, pp. 3028-3033.

[5] R. Liu, R. Chen, H. Shen, and R. Zhang, "Wall climbing robot using electrostatic adhesion force generated by flexible interdigital electrodes," Int. J. Adv. Robot. Syst., vol. 10, no. 36, pp. 1-9, 2012.

[6] B. Schubert and D. Floreano, "Stretchable electroadhesion for soft robots," in Proc. 2014 IEEE/RSJ Int. Conf. Intell. Robots Syst., 2014, pp. 3933-3938.

[7] K. H. Koh, M. Sreekumar, and S. G. Ponnambalam, "Hybrid electrostatic and elastomer adhesion mechanism for wall climbing robot," Mechatronics, vol. 35, pp. 122-135, 2016

[8] M. Graule et al., "Perching and takeoff of a robotic insect on overhangs using switchable electrostatic adhesion," Science, vol. 352, pp. 978-982, 2016

[9] Z. Zhang, "Modeling and analysis of electrostatic force for robot handling of fabric materials," IEEE/ASME Trans. Mechatronics., vol. 4, no. 1, pp. 39-49, Mar. 1999.

[10] J. Jin, T. Higuchi, and M. Kanemoto, "Electrostatic levitator for hard disk media," IEEE Trans. Ind. Electron., vol. IE-42, no. 5, pp. 467-473, Oct. 1995.

[11] J. Guo, M. Tailor, T. Bamber, M. Chamberlain, L. Justham, and M. Jackson, "Investigation of relationship between interfacial electroadhesive force and surface texture," J. Phys. D. Appl. Phys., vol. 49, no. 3, 2016, Art. no. 35303.

[12] J. Guo, L. Justham, M. Jackson, and R. Parkin, "A concept selection method for designing climbing robots," Key Eng. Mater, vol. 649, pp. 22-29, 2015.

[13] Sep. 2016. [Online]. Available: http://grabitinc.com/

[14] J. Guo, T. Bamber, M. Chamberlain, L. Justham, and M. Jackson, "Optimization and experimental verification of coplanar interdigital electroadhesives," J. Phys. D. Appl. Phys., vol. 49, no. 41, 2016, Art. no. 415304.
[15] D. Ruffatto, J. Shah, and M. Spenko, "Increasing the adhesion force of electrostatic adhesives using optimized electrode geometry and a novel manufacturing process," J. Electrostat., vol. 72, no. 2, pp. 147-155, 2014.

[16] J. Guo, T. Bamber, T. Hovell, M. Chamberlain, L. Justham, and M. Jackson, "Geometric optimisation of electroadhesive actuators based on 3D electrostatic simulation and its experimental verification," IFACPapersOnLine, vol. 49, no. 21, pp. 309-315, 2016.

[17] J. Krahn and C. Menon, "Electro-dry-adhesion," Langmuir, vol. 28, no. 12 , pp. 5438-5443, 2012.

[18] G. J. Monkman, "Compliant robotic devices, and electroadhesion," Robotica, vol. 10, no. 2, pp. 183-185, 1992.

[19] R. Dhelika, K. Sawai, K. Takahashi, W. Takarada, T. Kikutani, and S. Saito, "Electrostatic chuck consisting of polymeric electrostatic inductive fibers for handling of objects with rough surfaces," Smart Mater Struct., vol. 22, no. 9, 2013, Art. no. 095010.

[20] L. Savioli, G. Sguotti, A. Francesconi, F. Branz, J. Krahn, and C. Menon, "Morphing electroadhesive interface to manipulate uncooperative objects," Proc. SPIE, vol. 9061, 2014, Art. no. 906129.

[21] S. A. Suresh, D. L. Christensen, E. W. Hawkes, and M. Cutkosky, "Surface and shape deposition manufacturing for the fabrication of a curved surface gripper," J. Mech. Robot., vol. 7, no. 2, 2015, Art. no. 021005.

[22] H. Prahlad, T. P. Low, and R. E. Pelrine, "Vacuum augmented electroadhesive device," U.S. Patent 9130 484, 2014.

[23] J. Guo, T. Bamber, M. Chamberlain, L. Justham, and M. Jackson, "The influence of environmental factors on the electroadhesive forces obtainable during experimental testing," in Proc. IOP Early Career Researchers Colloq., London, U.K., 2015.

[24] G. J. Monkman, "An analysis of astrictive prehension," Int. J. Robot., vol. 16 , no. 1 , pp. $1-10,1997$.

[25] R. Liu, R. Chen, and H. Shen, "Modeling and analysis of electric field and electrostatic adhesion force generated by interdigital electrodes for wall climbing robots," in Proc. 2013 IEEE/RSJ Int. Conf. Intell. Robots Syst., 2013, pp. 2327-2332.

[26] J. Guo, "Numerical and experimental study of electroadhesion to enable manufacturing automation," Ph.D. dissertation, Wolfson School, Loughborough Univ., Loughborough, U.K., 2016. 